\title{
Multimodal Prostate Registration using Thin-Plate Splines from Automatic Correspondences
}

\author{
Jhimli Mitra*, Arnau Oliver, Robert Martí, Xavier Lladó \\ Computer Vision and Robotics Group, \\ University of Girona, \\ Campus Montilivi, Edifici P-IV, Av. Lluís Santaló, \\ s/n, 17071 Girona, Spain. \\ jhimlimitra@yahoo.com, (aoliver/marly/llado)@eia.udg.es \\ Fabrice Meriaudeau \\ *Laboratoire Le2I - UMR CNRS 5158, \\ Université de Bourgogne, \\ 12 Rue de la Fonderie, \\ 71200 Le Creusot, France. \\ fabrice.meriaudeau@u-bourgogne.fr
}

\author{
Joan C. Vilanova \\ Clinica Girona, \\ Calle Joan Maragall 26, \\ 17002 Girona, Spain. \\ kvilanova@comg.cat
}

\begin{abstract}
Registration is an important step during prostate biopsy with interventional Ultrasound (US) to project the Magnetic Resonance (MR) information of the corresponding slice of a pre-acquired MR volume of the same patient. This paper presents a method of deformable registration between US and MR prostate modalities using Thin-Plate Splines (TPS). The use of radial-basis functions such as splines is a typical approach for deformable registration wherein, TPS are more computationally efficient than $B$ splines. However, registration with TPS employs strategic choice of correspondence points in the moving and reference images and therefore, has been rarely used by researchers for prostate registration. Aiming to deal with this issue, a novel method to automatically choose correspondence points (control points) based on the prostate geometry is presented in this paper. Shepard's interpolation is used with the TPS in order to deal with the interpolation artifacts produced by reverse TPS transform. To evaluate the accuracy of our correspondence generation method, two intuitive geometric methods for prostate contour sampling are also implemented. Our registration results are validated using 4 patient datasets obtaining an average DSC of $0.97 \pm 0.01$.
\end{abstract}

\section{Introduction}

Prostate biopsy is often performed with interventional Trans-Rectal Ultrasound (TRUS) imaging. Ultrasound images provide a satisfactory boundary of the prostate without any contrast between the normal and malignant tissues of the prostate. Therefore, clinicians generally extract 10-12 samples strategically from different regions of the prostate. This approach increases the risk of malignant regions not being uncovered and consequently the number of re-biopsies [9]. However, malignant prostate regions may be detected more accurately using MR imaging. MR images provides a better contrast between the normal and malignant tissues of the prostate. Therefore, informations from MR images need to be fused with the interventional US images of the same patient [5, 2, 13, 12,7]. Deformable registration is necessary for the fusion of interventional US and pre-acquired MR images as the prostate may undergo significant deformations due to the insertion of the endorectal probe through the rectum during the MRI, inflation of the endorectal balloon, full bladder, bowel and gas in rectum or different patient positions on the couch during MRI and US procedures.

Deformable registration using TPS requires selection of correspondence points on the moving and reference images and the transformation parameters are computed from these correspondences. Lu et al. [6] and Fei et al. [3] used TPS with manually chosen correspondences to warp MR prostate volumes. Salient points on prostate contour 
were automatically identified using equal angle sampling by Padilla-Castañeda and Arámbula-Cosío [8]. Yan et al. [14] used equally spaced salient points on prostate contour based on Euclidean distance to build a partial active shape model for segmentation.

This paper focuses on deformable multimodal prostate registration employing TPS and proposes a novel approach to establish correspondence points in the US and MR modalities. Our approach does not require correspondences to be established manually and is fully automatic after obtaining the segmented prostate contours. The generation of correspondence points exploits the shape geometry of the prostate and its principal shape components. Thereafter, correspondence points are generated in different resolutions. Resolution signifies sparse to dense set of control points for TPS. The TPS framework includes the correspondence localization errors and uses inverse-square distance weighted commonly known as Shepard's interpolation [11] to yield a smooth transformation. The accuracy of our correspondence point generation algorithm for registration is evaluated against two intuitive geometric prostate contour sampling methods. The results are validated on 4 patients, where, our method of generating correspondences always outperforms the other geometric methods.

The organization of the paper is as follows: section 2 describes the methods implemented, section 3 compares the results of different correspondence points generation algorithms along with the improved registration quality using Shepard's interpolation over reverse TPS interpolation. Conclusions and future improvements are presented in section 4.

\section{Proposed method}

This section is divided into two subsections; the first one dealing with the correspondence points generation algorithm and the second one explaining the thin-plate splines with Shepard's interpolation.

\subsection{Automatic correspondences}

Assuming that the 2D MR/US corresponding slices are found on visual inspection by an expert, our correspondence points generation algorithm is based on a triangulation method with the principal axes of the segmented prostate as the underlying framework. Principal axes make the best representation of the primary orientation of a shape and are generated from the Principal Component Analysis (PCA) of the contour points of the shape.

The 2D-US prostate reference image and the corresponding 2D-MR moving image from the MR volume are initially segmented using the method of Ghose et al. [4]. Principal axes of the reference image are generated and projected onto the moving image with shifted mean assum- ing that the corresponding slices have no rotation of the prostate between them. The intersection points of the principal axes with the prostate boundary for both the reference and moving images are identified automatically. The triangulation method to generate correspondence points begins by traversing the intersections of the principal axes in a clockwise or anti-clockwise manner in each image. The order of traversing should however be the same for both the reference and moving images.

Triangles are generated by joining the adjacent intersections of the principal axes forming a quadrant and dropping a perpendicular from the midpoint of the line joining these intersections. The adjacent intersection points and the point of intersection of the perpendicular dropped on the prostate contour comprise a triangular approximation of the prostate region in the quadrant. Likewise, other quadrants of the shape are processed for both the reference and moving images. Hence, new points formed by the triangulation method are generated on the shape contours for the first resolution (see Fig. 1(b)). Resolution signifies coarser to denser control points on the shape contour. The points are reordered and further triangulations are made at subsequent resolutions, approximating smaller prostate regions close to the boundary. Fig. 1 shows the triangulation method for three subsequent levels.

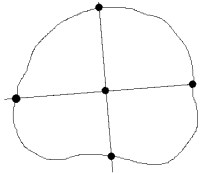

(a) Level 0

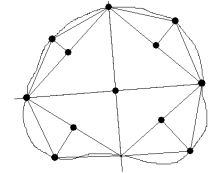

(b) Level

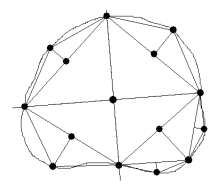

(c) Level 2, only shown for right-
bottom quadrant
Figure 1. Method of generating correspondence points in different resolutions.

Instead of considering only the contour points for the deformable registration, certain points inside the prostate contour are also considered for a smooth deformation of the internal glandular structures of the prostate that are quite evident in MR images and sometimes partially visible in US images. The internal points are primarily the prostate centroid and the midpoints of the line joining the adjacent principal axes in the first resolution (see Fig. 1(b) and 1(c)).

Two methods of prostate contour sampling at equal angles and equal spaces are also implemented to compare the accuracies of our proposed correspondence generation method. These geometric sampling methods are intuitive where, in equal-angle sampling, the angles are equally spread inside each quadrant formed by the principal axes. In equal-space contour sampling, the points are also generated inside each quadrant depending on the number of contour points falling in the same. Fig. 2(a) and Fig. 2(b) 


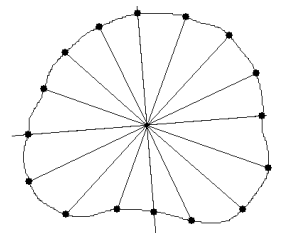

(a) Equal-angle sampling

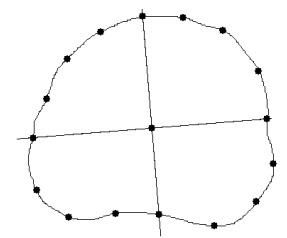

(b) Equal-space sampling
Figure 2. Geometric methods for correspondence points generation.

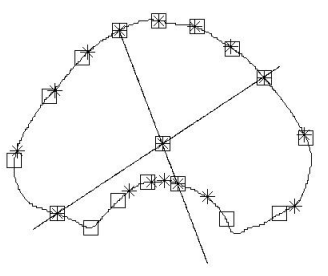

(a) Points generated in US

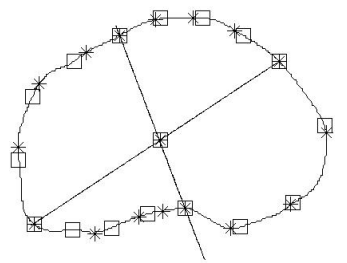

(b) Points generated in MR
Figure 3 . The $*_{s}$ indicate points generated with equal-angle sampling and the squares indicate the points generated with our approach. Note thhat in bottom-left quadrant with our method, we are able to get good correspondences even in the presence of a significant deformation.

show the contour points obtained with equal-angle sampling and equal-space sampling approaches respectively. Fig. 3 shows a case when bad correspondences are generated with equal-angle sampling due to significant deformation between the reference and moving images. Our method makes triangular approximations of prostate quadrants and smaller regions and moves towards the prostate boundary in a symmetric manner with the perpendicular bisector of the triangle as reference. Other methods use the prostate centroid only as the reference lying far from the boundary and therefore the localization error of correspondences increases.

\subsection{Thin-plate splines registration}

The thin-plate spline is a commonly used basis function in 2D-Euclidean space [1] to map the coordinates of a moving image into a reference image, when a set of homologous correspondence points are established in both images. In its regularized form, the deformable TPS model includes the affine model as a special case. Correspondences are generated automatically on both the moving and reference images. Following is a brief algebraic description of the TPS model.

If $p_{i}=\left(x_{i}, y_{i}\right)$ and $q_{i}=\left(x_{j}, y_{j}\right), i=j=1, \ldots, n$ represent two sets of corresponding landmarks in the moving and reference images respectively, then, the TPS interpolant $f(x, y)$ minimizes the bending energy or the integral bending norm

$$
I_{f}=\iint_{\Re^{2}}\left(f_{x x}^{2}+2 f_{x y}^{2}+f_{y y}^{2}\right) d x d y
$$

and has the form

$$
\begin{aligned}
& f(x, y)=a_{1}+a_{x} x+a_{y} y+ \\
& \sum_{i=1}^{n} w_{i} U\left(\left\|\left(x_{i}, y_{i}\right)-(x, y)\right\|\right),
\end{aligned}
$$

where, $U=r^{2} \log r . f(x, y)$ should have square integrable derivatives if

$$
\sum_{i=1}^{n} w_{i}=0 \quad \text { and } \quad \sum_{i=1}^{n} w_{i} x_{i}=\sum_{i=1}^{n} w_{i} y_{i}=0
$$

Together with the interpolation conditions, $f\left(x_{i}, y_{i}\right)=q_{i}$, a linear system is obtained for the TPS coefficients as

$$
\left[\begin{array}{cc}
K & P \\
P^{T} & O
\end{array}\right]\left[\begin{array}{l}
w \\
a
\end{array}\right]=\left[\begin{array}{l}
q \\
o
\end{array}\right]
$$

where, $K$ is a $n \times n$ matrix and $K_{i j}=$ $U\left(\left\|\left(x_{i}, y_{i}\right)-\left(x_{j}, y_{j}\right)\right\|\right)$, $i$ th row of $P$ is $\left(1, x_{i}, y_{i}\right)$, $O$ is a $3 \times 3$ matrix of zeros, $o$ is a $3 \times 1$ column vector of zeros, $w$ and $q$ are column vectors of $w_{i}$ s and $q_{j}$ s respectively and $a$ is a column vector of the affine parameters $a_{1}$, $a_{x}$ and $a_{y}$.

Localization errors of the correspondence points may be considered by extending the interpolation to regularization [10]. This is accomplished by the minimization of

$$
H(f)=\sum_{i=1}^{n} \frac{\left(q_{i}-f\left(x_{i}, y_{i}\right)\right)^{2}}{\sigma_{i}^{2}}+\lambda I_{f}
$$

The covariance $\sigma_{i}^{2}$ is the sum of the covariances of the points $p_{i}$ and $q_{i}$ and $\lambda=0.01$ is the regularization term. This minimization can be analytically formed into an equation

$$
\left[\begin{array}{cc}
K+n \lambda C^{-1} & P \\
P^{T} & O
\end{array}\right]\left[\begin{array}{l}
w \\
a
\end{array}\right]=\left[\begin{array}{l}
q \\
o
\end{array}\right]
$$

where,

$$
C^{-1}=\left(\begin{array}{ccc}
\sigma_{1}^{2} & & 0 \\
& \ddots & \\
0 & & \sigma_{n}^{2}
\end{array}\right)
$$

Introducing the term $n \lambda C^{-1}$ yields a better conditioned linear system and a robust numerical solution. Finally, (5) is framed as

$$
L U=V
$$

and solved as

$$
U=L^{-1} V
$$


where,

$$
L=\left[\begin{array}{cc}
K+n \lambda C^{-1} & P \\
P^{T} & O
\end{array}\right], U=\left[\begin{array}{l}
w \\
a
\end{array}\right]
$$

and

$$
V=\left[\begin{array}{l}
q \\
o
\end{array}\right]
$$

The TPS and affine parameters obtained from the TPS framework are used to warp the moving image using nearest-neighbor interpolation to map the pixel values onto integer coordinates. However, TPS interpolation produces holes in the transformed image as not all the pixels are being mapped onto it. Therefore, a general approach is to unwarp the transformed image to fill up the unmapped pixels. Unfortunately, the reverse transformation fails to achieve accurate results since, splines are not exactly reversible. Hence, we propose to use Shepard's interpolation [11] to fill the holes produced by forward TPS interpolation. This method of interpolation successfully interpolates a point from scattered pixel points. In our implementation, an unmapped pixel is mapped with an intensity value, interpolated from the scattered mapped pixels in a $7 \times 7$ mask centering the pixel. This interpolation method is faster than computing the reverse TPS parameters and produces smooth intensity warping of the moving image.

\section{Results and Discussions}

Our proposed methods are tested with 4 patients' middle slice images of the prostate from which, automatic prostate segmentations are available in both US (Siemens ACUSON) and MR images (GE 1.5T). For all the images we applied the three correspondence generation methods described in section 2.1. Fig. 4 shows the obtained results with Patient-1. Observe that our correspondence points (see last row of Fig. 4), yield better registration result than the other two approaches. The number of correspondences generated using our method normally starts from level 2 including the center and other internal points i.e. 21 correspondences. It is observed that a maximum of 37 correspondences (level 3 ) are required for a smooth warping.

Notice from columns 1 and 2 of Fig. 4 that different numbers of correspondences are generated for each approach as these are found to be optimal to provide satisfactory registration qualities. With our method 37 correspondences yields a smooth deformation. On the other hand, with other methods less number of correspondences provide satisfactory results. However, increase in number of points in the latter methods increases correspondence localization errors and hence yield poor registrations. This is well justified with the fact that our approach estimates correspondence points in localized regions and thus, can avoid undesirable localization errors.
Observing Fig. 4 (1st and 2nd rows) note that overall accuracies of the registration along the prostate boundaries are satisfactory, when equal-angle and equal-space samplings are used respectively, although the internal structures do not have smooth deformations. Equal-angle sampling produces better results for Patients 1 and 3 and produce undesirable registrations for Patients 2 and 4. Therefore, only Patient-1 results with all the correspondence point generation methods have been presented to maintain the brevity of the paper. Fig. 5 shows the results obtained with our correspondence approach for patients 2, 3 and 4 .

Registration accuracy is evaluated by computing the Dice Similarity Coefficient (DSC), measuring the overlap between the transformed moving image and the reference image. Table 1 shows DSC values obtained with our approach along with the computation times for generating correspondence points and TPS interpolation with Shepard's interpolation. An average DSC of $0.97 \pm 0.01$ is obtained for all the patients. The DSC values after TPS registration using the equal-angle sampling and equal-space sampling and our correspondence method only for Patient- 1 are 0.97 and 0.96 and 0.98 respectively. Therefore, it may be concluded that although, equal-angle sampling and equal-space sampling correspondence methods provide high DSC values in certain cases, specially when the prostate contours have similar shapes in the respective modalities, our approach always outperforms the other geometric approaches.

Fig. 6 shows the interpolation artifacts in the deformed image when reverse TPS is used (Fig. 6(a)) and a smooth deformation when Shepard's interpolation method is applied (Fig. 6(b)). The algorithms have been implemented in MATLAB with $1.66 \mathrm{GHz}$, Core2Duo processor with 2GB memory and it has been observed that a forward TPS and Shepard's interpolation is more computationally efficient than forward and reverse TPS interpolation.

\section{Conclusions and Future Works}

A novel geometric approach to generate correspondence points for registration, based on segmented prostate contour has been presented. The method is based on triangular approximation of closed boundary regions that may be generalized for regular-shaped anatomical structures. Our method showed better correspondences being generated, resulting in smooth deformations and significantly high region overlaps after registration, in comparison with other geometric correspondence points generation methods. The method is computationally efficient in order to be used during biopsy procedures. TPS interpolation along with Shepard's interpolation produced smoother deformations and could avoid interpolation artifacts resulting from reverse TPS interpolation.

We propose to validate our method of correspondence point generation with more patient data and claim on the 

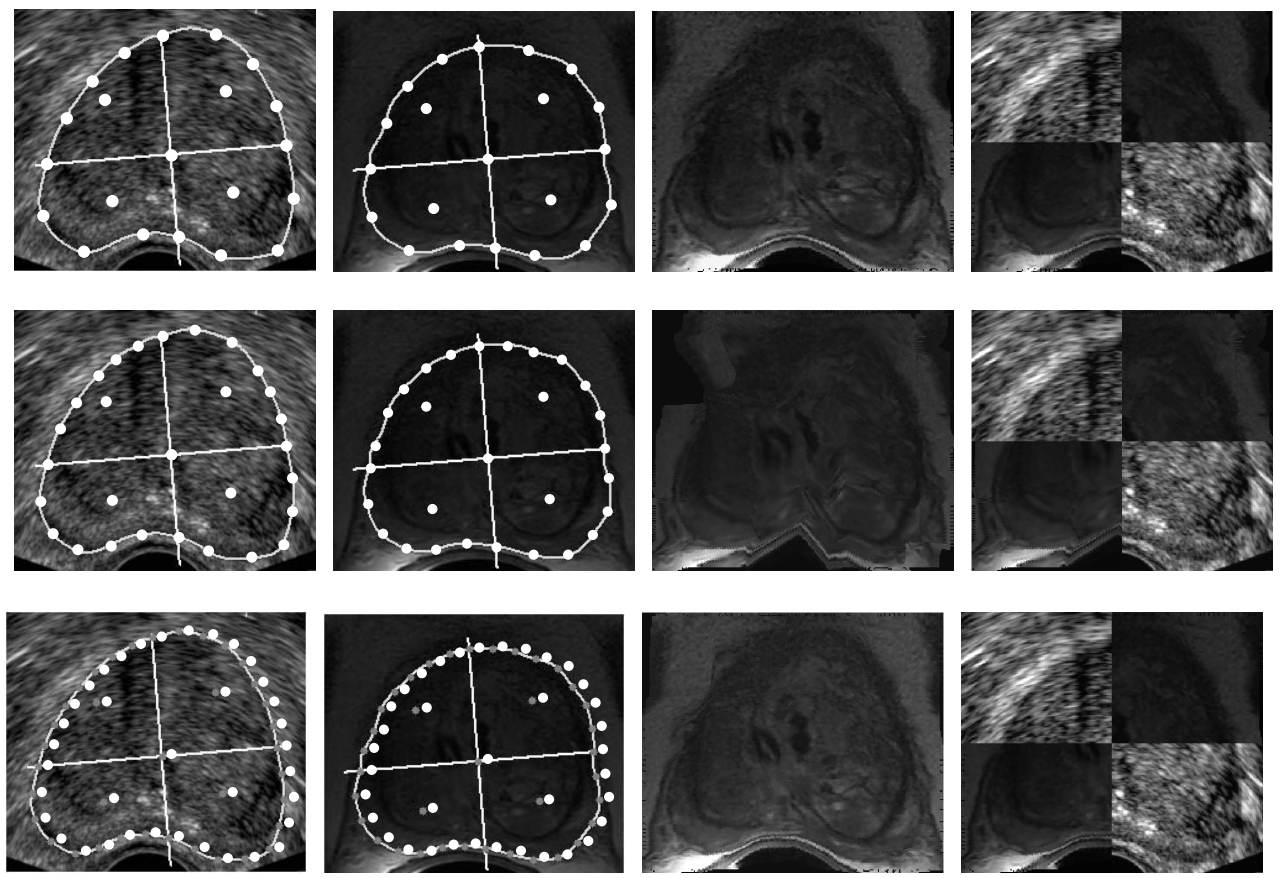

Figure 4. Qualitative registration results for Patient-1. (First-row) correspondences using equal-angle sampling,(second-row) correspondences using equal-space sampling, (third-row) correspondences using our method. The first-left column shows the reference US images, second column shows the moving MR images, third column consists of the registered images and the last column consists of checker-board displays to evaluate quality of registration.
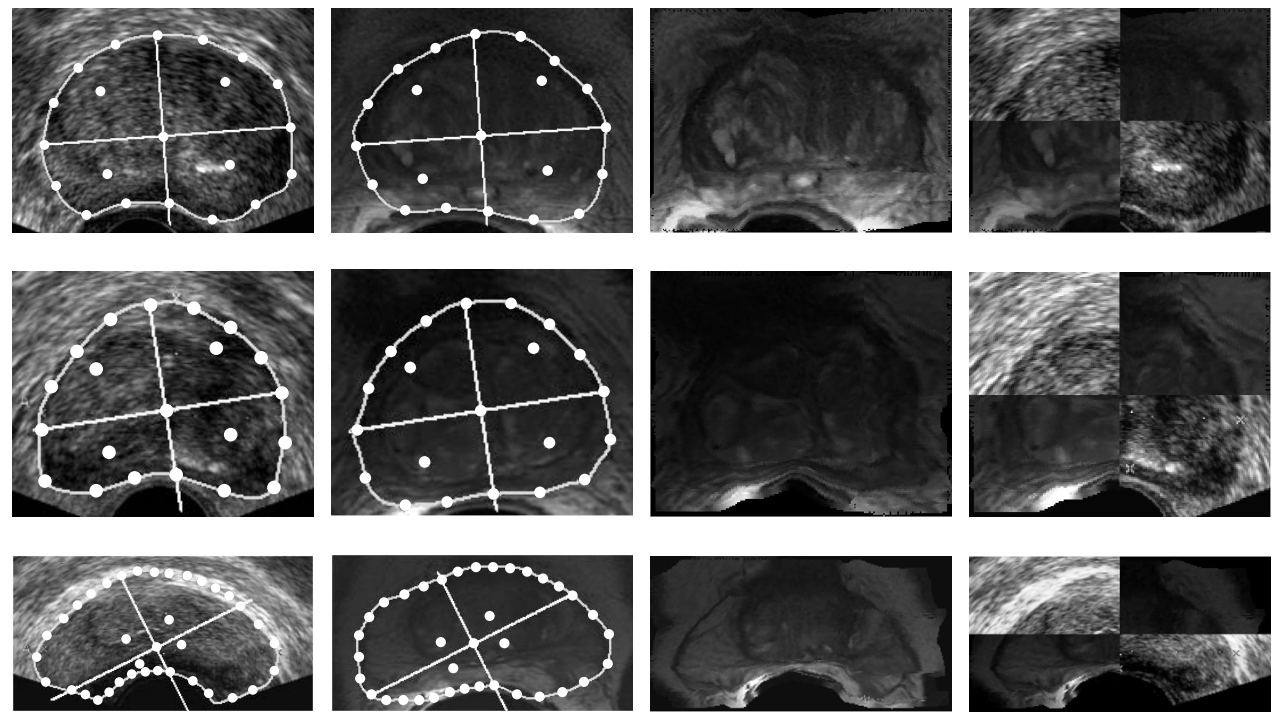

Figure 5. Patient-2 to Patient-4 registration results using our method of correspondences. The columns are similar to Fig. 4.

robustness of the algorithm. We also propose to extend our registration framework to the fusion of 3D TRUS/MR volumes and develop a method to search for the corresponding US and MR slices automatically. Optimal number of correspondences could be generated incorporating mutual information maximization in the TPS framework. The maximum time during TPS interpolation is required in transforming the moving image with the interpolation parameters, rather than the optimization phase that requires less than 1 sec. Therefore, the forward TPS with Shepard's interpolation can be accelerated if the transformation is parallelized with GPU implementation. Conclusively, our method of generating correspondences and the TPS/Shepard's interpolation are efficient and may be used for non-rigid registration during US interventional biopsies if the intermediate manual interventions are removed and the implementation is paral- 
Table 1. Computational performance and accuracy of the proposed algorithm.

\begin{tabular}{llll}
\hline \hline Patient\# & $\begin{array}{l}\text { Correspondence } \\
\text { points method(secs) }\end{array}$ & $\begin{array}{l}\text { TPS/Shepard's } \\
\text { method(secs) }\end{array}$ & DSC \\
\hline 1 & 0.2 & 94.58 & 0.98 \\
2 & 0.2 & 83.91 & 0.97 \\
3 & 0.14 & 22.71 & 0.96 \\
4 & 0.16 & 50.15 & 0.97 \\
\hline
\end{tabular}

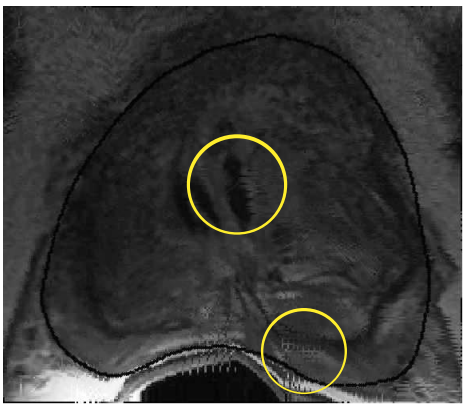

(a) Forward-Reverse TPS interpolation

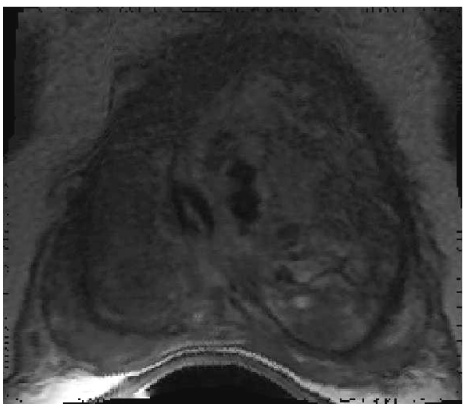

(b) Forward TPS/Shepard's interpolation

Figure 6. Comparison of forward-reverse TPS interpolation with forward TPS/Shepard's interpolation, (a) interpolation artifacts visible in center and lower part of the image (encircled), (b) smooth deformation.

lelized.

\section{References}

[1] F. Bookstein. Morphometric Tools for Landmark Data: Geometry and Biology. Cambridge University Press, Cambridge, UK, 1991.

[2] V. Daanen, J. Gastaldo, J. Y. Giraud, P. Fourneret, J. L. Descotes, M. Bolla, D. Collomb, and J. Troccaz. MRI/TRUS data fusion for brachytherapy. Intl. Journal of Medical Robotics and Computer Assisted Surgery, 2(3):256-261, 2006.

[3] B. Fei, C. Kemper, and D. L. Wilson. A comparative study of warping and rigid body registration for the prostate and pelvic MR volumes. Computerized Medical Imaging and Graphics, 27:267-281, 2003.

[4] S. Ghose, A. Oliver, R. Martí, X. Lladó, J. Freixenet, J. Vilanova, and F. Meriaudeau. Texture guided Active Appearance Model propagation for prostate segmentation. Accepted in Prostate Cancer Imaging, Computer Aided Diagnosis, Prognosis and Intervention, MICCAI workshop, 2010.

[5] I. Kaplan, N. E. Oldenburg, P. Meskell, M. Blake, P. Church, and E. J. Holupka. Real time MRI-ultrasound image guided stereotactic prostate biopsy. Magnetic Resonance Imaging, 20:295-299, 2002.

[6] J. Lu, R. Srikanchana, M. McClain, Y. Wang, J. Xuan, I. A. Sesterhenn, M. T. Freedman, and S. K. Mun. A statistical volumetric model for characterization and visualization of prostate cancer. Proc. of SPIE, 3976:142-153, February 2000.

[7] R. Narayanan, J. Kurhanewicz, K. Shinohara, E. D. Crawford, A. Simoneau, and J. S. Suri. MRI-ultrasound registration for targeted prostate biopsy. IEEE Intl. Symposium on
Biomedical Imaging: From Nano to Macro, pages 991-994, 2009.

[8] M. A. Padilla-Castañeda and F. Arámbula-Cosió. Resection simulations with local tissue deformations for computer assisted surgery of the prostate. MICAI, LNAI, 2313:450-459, 2002.

[9] C. R. Porter, C. O’Donnell, E. D. Crawford, E. J. Gamito, J. Kim, and A. Tewari. Predicting the outcome of the random prostate biopsy. Technical report, Department of Urology, State University of New York, Stony Brook,NY. http://www.cancernews.com/data/Article/230.asp, accessed [30 May 2010].

[10] K. Rohr, H. S. Stiehl, R. Sprengel, T. M. Buzug, J. Weese, and M. H. Kuhn. Landmark-based elastic registration using approximating thin-plate splines. IEEE Transactions on Pattern Analysis and Machine Intelligence, 20(6), June 2001.

[11] D. Shepard. A two-dimensional interpolation function for irregularly-spaced data. Proceedings of the 1968 23rd ACM national conference, pages 517-524, 1968.

[12] A. K. Singh, J. Kruecker, S. Xu, N. Glossop, P. Guion, K. Ullman, P. L. Choyke, and B. J. Wood. Initial clinical experience with real-time transrectal ultrasonographymagnetic resonance imaging fusion-guided prostate biopsy. British Journal of Urology (BJU) International, 101(7):841845, April 2008.

[13] S. Xu, J. Kruecker, B. Turkbey, N. Glossop, A. K. Singh, P. Choyke, P. Pinto, and B. J. Wood. Real-time MRI-TRUS fusion for guidance of targeted prostate biopsies. Computer Aided Surgery, 13(5):255-264, 2008.

[14] P. Yan, S. Xu, B. Turkbey, and J. Kruecker. Optimal search guided by partial active shape model for prostate segmentation in trus images. Proceedings of SPIE Medical Imaging 7261:72611G.1-72611G.11, 2009. 